\title{
Glycolysis and Its Interaction with a Gluconeogenic Precursor in Perfused Rat Liver*
}

\author{
W. Hansen and P. Bottermann \\ 2. Medizinische Klinik der Technischen Universität München, Munich, Federal Republic of Germany
}

Received: September 26, 1974, and in revised form: June 27, 1975

\begin{abstract}
Summary. A non-recirculating, haemoglobin-free perfusion system was used to evaluate glycolysis in livers from fasted rats. Glycolysis was calculated from lactate and pyruvate production before and after infusion of glucose. In the absence of extracellular glucose glycolysis was small. Stimulation of glycolytic flux occured at extracellular glucose concentrations above $5 \mathrm{mM}$. Rates were proportional to glucose concentrations. Glycolysis was also investigated in the presence of extracellular lactate, which is a precursor of gluconeogenesis. High glucose concentrations (15-35
\end{abstract}

$\mathrm{mM}$ ) were followed by lactate and pyruvate output suggesting that glycolysis was prevailing. Estimated flux rates, however, were significantly lower in the presence of the gluconeogenic precursor. Our results provide arguments that gluconeogenesis and glycolysis interact in order to regulate the peripheral glucose concentration.

Key words: Gluconeogenesis, glucose, glycolysis, lactate, liver, pyruvate.
Glycolysis has been demonstrated in rat liver slices [1] and isolated rat liver cells [2] only at minor rates. It therefore appeared questionable, whether the "liver under aerobic conditions ever exhibits a net carbohydrate flow in the direction of glucose catabolism" [3]. On the other hand it has been shown in man that infusion of glucose can lead to splanchnic lactate production [4]. Administration of glucose to rats resulted in enhanced hepatic lipogenesis [5]. Both observations could be explained by significant glycolytic breakdown of glucose in liver under physiological conditions.

The following investigations were designed to reevaluate the former controversial findings on glycolysis with a non-recirculating liver-perfusion system [6]. Disturbances from erythrocyte metabolism and interference from hormones were avoided by the use of a haemoglobin-free Krebs-Henseleit bicarbonate medium. The aim was to provide a basis for further understanding the liver's contribution to glucose metabolism.

\section{Materials and Methods}

Materials and general techniques have been described elsewhere [7]. Livers from male albino rats (Wistar strain, 150-180 g) fasted for $24 \mathrm{hrs}$ were

\footnotetext{
* The results were presented in part at the $9^{\text {th }}$ Congress of the German Diabetes Association, Travemünde, 1974.

Dedicated to Professor Dr. Hans Ley on his 60th birthday.
}

perfused with a haemoglobin-free Krebs-Henseleit bicarbonate medium in a non-recirculating system. However, a modified membrane oxygenator was employed which enabled complete saturation with $95 \% \mathrm{O}_{2}$ and $5 \% \mathrm{CO}_{2}$ (W. Hansen, unpublished). Oxygen concentration was monitored continuously from the outflowing perfusate employing a teflonshielded platinum electrode. Oxygen-uptake was calculated from the a-v difference on the basis of an arterial oxygen saturation of $95 \%$. For statistical calculations Student's t-test was used. - In all instances reagent grade chemicals were used. Glucose was obtained from Merck, Darmstadt; sodium $(+)$-lactate was purchased from Roth, Karlsruhe. Glucose, lactate and pyruvate were analyzed by standard enzymatic procedures [8].

\section{Results}

\section{Effect of Increasing Extracellular Glucose Con- centrations}

A typical experiment in which various glucose concentrations were infused is represented in Fig. 1. During $20 \mathrm{~min}$ of preperfusion little lactate and pyruvate could be detected in the outflowing perfusate. Addition of $4 \mathrm{mM}$ glucose resulted in no change in oxygen consumption and practically no stimulation of lactate and pyruvate production. This could be explained by the fact that at $4 \mathrm{mM}$, which corresponds to a normal blood glucose value, there was no net uptake of 
glucose $[9,10]$. Elevation of glucose concentrations to 12,24 and $40 \mathrm{mM}$ in a stepwise manner, however, was followed by a concomitant increase in lactate and pyruvate production rates indicating that glucose was taken up and metabolized via the Embden-Meyerhof pathway. At 12, 24 and $40 \mathrm{mM}$ there was also an increased oxygen consumption suggesting that processes requiring energy were stimulated, e.g. glycogen synthesis $[11,12]$. When the infusion of glucose was terminated all rates returned to the original values. There was temporary glucose production, possibly from break-down of newly formed glycogen (not shown in Fig. 1).

The quantitative relationship between extracellular glucose concentrations and lactate and pyruvate production rates is shown in Table 1.

\section{Glycolysis in the Presence of Extracellular Lactate}

Under physiological conditions in blood compounds besides glucose can be found which are substrates for gluconeogenesis. Glycolysis is likely to interfere with gluconeogenesis since antagonistic enzymatic reactions are involved. In order to further elucidate the significance of glycolytic break-down of glucose in liver the interaction with a gluconeogenic precursor was investigated. Lactate was chosen as a model substrate. A typical experiment is represented in Fig. 2.
Infusion of $0.4 \mathrm{mM}$ lactate resulted in gluconeogenesis as seen by uptake of lactate and an increased oxygen consumption. It has been shown previously that under these conditions the stoichiometry of lactate uptake to glucose production is nearly two to one [12]. Infusion of $5 \mathrm{mM}$ glucose did neither influence lactate uptake, pyruvate output nor did it influence oxygen consumption significantly, suggesting that gluconeogenesis was not altered. Further increase of glucose concentrations to 15 and $35 \mathrm{mM}$, however,

Table 1. Dependence of rates of lactate and pyruvate production on the glucose concentration. Data are from experiments similar to that in Fig. 1. Results are given as mean \pm SEM. Glucose concentrations above $5 \mathrm{mM}$ resulted in increased lactate and pyruvate output. The rates were proportional to the concentration of the infused glucose, their differences were statistically significant $(p<0.01)$

\begin{tabular}{llcc}
$\begin{array}{l}\text { Extracellular } \\
\text { glucose concen- } \\
\text { tration }(\mathrm{mM})\end{array}$ & $\begin{array}{l}\text { Lactate } \\
\text { output } \\
\left(\mu \mathrm{mol} \cdot \mathrm{g}^{-1} \cdot \mathrm{h}^{-1}\right)\end{array}$ & $\begin{array}{l}\text { Pyruvate } \\
\text { output } \\
\left(\mu \mathrm{mol} \cdot \mathrm{g}^{-1} \cdot \mathrm{h}^{-1}\right)\end{array}$ & $\begin{array}{l}\text { Nr. of } \\
\text { obser- } \\
\text { vations }\end{array}$ \\
\hline 0 & $4.6 \pm 0.5$ & $0.3 \pm 0.2$ & 25 \\
5 & $5.1 \pm 0.8$ & $0.4 \pm 0.2$ & 9 \\
7.5 & $18.4 \pm 3.6^{\mathrm{a}}$ & $1.6 \pm 0.3^{\mathrm{a}}$ & 9 \\
10 & $29.5 \pm 3.1^{\mathrm{a}}$ & $3.8 \pm 0.2^{\mathrm{a}}$ & 5 \\
15 & $58.5 \pm 4.9^{\mathrm{a}}$ & $17.3 \pm 2.7^{\mathrm{a}}$ & 5 \\
\hline
\end{tabular}

a $\mathrm{p}<0.01$

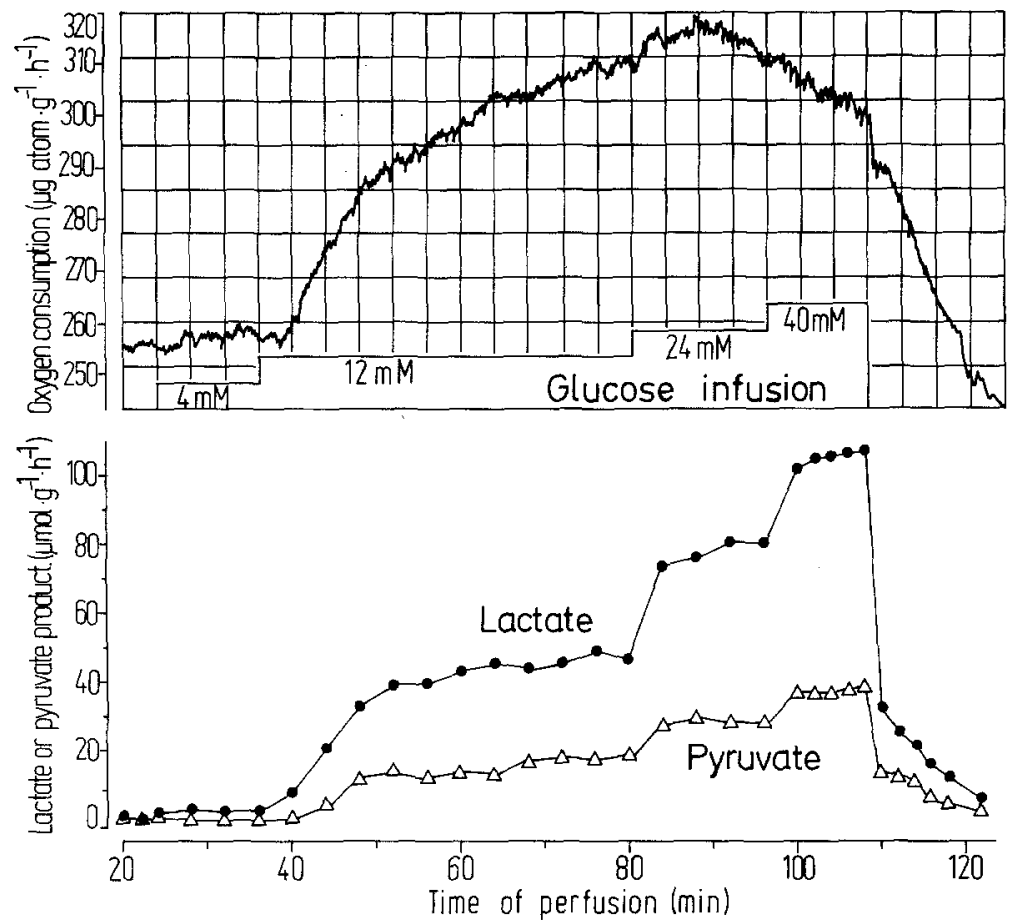

Fig. 1. Glycolysis from glucose in perfused liver of a fasted rat. Effect of increasing glucose concentrations on oxygen consumption (above), lactate and pyruvate production (below). The liver was perfused with non-recirculating Krebs-Henseleit bicarbonate buffer. Infusion of glucose is indicated by horizontal bars with numbers representing the concentrations entering the liver 
resulted in a reversal of the lactate balance indicating that glycolytic breakdown of glucose was the dominating process now. When the infusions of glucose and lactate were terminated rates returned to original values.

An exact calculation of gluconeogenesis and glycolysis is difficult to perform in this kind of experimental set-up since the changes in the extracellular glucose-concentrations are too small to be detected. Carbon flux, however, can be roughly estimated from the a-v differences in lactate and pyruvate concentrations, if one assumes that gluconeogenesis and glycolysis are the main processes involved. Thus uptake of lactate would indicate gluconeogenesis, output of lactate would indicate glycolysis. In both conditions production of pyruvate occured. For the evaluation of $\mathrm{C}_{3}$-flux in glycolysis we assumed that the pyruvate production came from glucose catabolism and added lactate and pyruvate production rates. In gluconeogenesis we assumed that the pyruvate output was derived from the infused lactate and subtracted pyruvate production rate from lactate uptake rate. Results so obtained are presented in Table 2. Glycolysis as reflected by $\mathrm{C}_{3}$-flux was significantly lower in the presence of the gluconeogenic precursor. The molar ratio lactate/pyruvate in the outflowing perfusate was lower in glycolysis (3.5); in gluconeogenesis it averaged 10.3. In the presence of glucose and lactate at comparable concentrations the ratio was 5.7 .

\section{Discussion}

In these studies lactate and pyruvate production was used to estimate glycolytic flux before and after glucose infusion. It was demonstrated in the glycogendepleted isolated perfused rat liver that under aerobic conditions hyperglycemia leads to significant stimulation of glycolysis. The rate of lactate and pyruvate production was proportional to the concentration of glucose infused (Table 1). From this finding it is tempting to speculate that glucose catabolism via the Emden-Meyerhof pathway may be a tool for lowering blood glucose concentration, a fact that has been questioned so far $[2,3]$. For full understanding of the physiological significance of this, however, it remains to be established how far other factors, e.g. hormones, exert control.
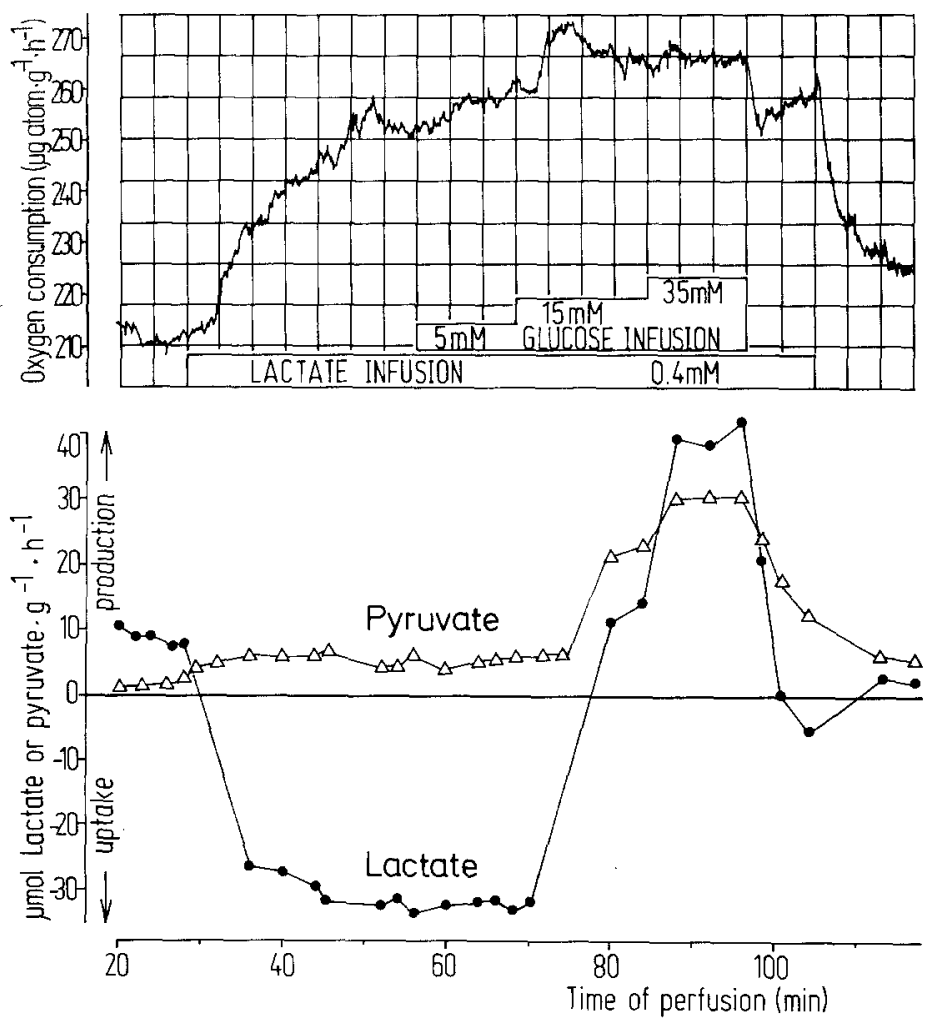

Fig. 2. Gluconeogenesis from lactate by perfused liver of a fasted rat. Effect of increasing glucose concentrations on lactate balance, pyruvate production and oxygen consumption. Infusion of lactate and glucose is indicated by horizontal bars with numbers representing concentrations in the perfusion fluid entering the liver. Production rates of lactate were calculated from the difference in concentration entering and leaving the liver 
Table 2. Effect of lactate and/or glucose infusion upon carbon flux and lactate/pyruvate ratio in perfused livers of fasted rats. Lactate uptake was calculated from the a-v differences and the perfusate's flow; the pyruvate output was subtracted. $C_{3}$-production was calculated as the sum of lactate and pyruvate production rates. There was no difference in the concentrations of the infused lactate or glucose. $C_{3}$-flux and lactate/pyruvate ratios showed statistically significant differences $(p<0.01)$

\begin{tabular}{|c|c|c|c|c|}
\hline $\begin{array}{l}\text { Infused } \\
\text { Lactate } \\
(\mathrm{mM})\end{array}$ & $\begin{array}{l}\text { bstrates } \\
\text { Glucose } \\
(\mathrm{mM})\end{array}$ & $\begin{array}{l}c_{3}-\text { flux } \\
\left.\text { (jumoles } \times g \text { liver }^{-1} \times h^{-1}\right)\end{array}$ & $\begin{array}{c}\text { Molar ratio } \\
\text { loctate / pyrumate } \\
\text { in outflowing perfusate }\end{array}$ & $\begin{array}{l}\text { Number } \\
\text { of } \\
\text { experinents }\end{array}$ \\
\hline $0.7 \pm 0.1$ & - & $-79 \pm 15$ & $10.3 \pm 0.3$ & 6 \\
\hline $0.7 \pm 0.2$ & $24 \pm 4$ & $+51 \pm 9$ & $5.7 \pm 0.9$ & 5 \\
\hline - & $26 \pm 4$ & $+101 \pm 11$ & $3.5 \pm 0.1$ & 7 \\
\hline
\end{tabular}

The estimated $\mathrm{C}_{3}$-flux was significantly lower in the presence of lactate, a precursor of gluconeogenesis (Table 2). From theoretical considerations there may be some objections to this finding, since it is based upon measurements outside the liver cells and it is not known how far this reflects intracellular processes. Possible variations in the metabolic activity between liver cells or liver lobules are not considered. Also the results are only true if one assumes that gluconeogenesis and glycolysis are the main processes involved. For instance pyruvate decarboxylation cannot be ruled out; however, it may be quantitatively small in glycolysis since pyruvate is washed out easily by the high perfusate flow, as can be seen from the high production rates. Moreover, it has been shown previously that in gluconeogenesis from lactate, pyruvate decarboxylation is so small that it can be neglected [7]. The reason why we performed these experiments was an interesting speculation on the regulation of glucose metabolism: it has been shown that gluconeogenesis from lactate [13] and alanine [14] is inhibited by high glucose concentrations. Together with our findings it seems possible that glycolysis and gluconeogenesis may be counteracting cellular reactions for the regulation of blood glucose concentration; if the extracellular glucose concentration is below normal gluconeogenesis will dominate in order to increase the blood glucose level; in the presence of high glucose concentrations glucose will be taken up and glycolysis will dominate, e.g. for lipid formation. From this point of view gluconeogenesis and glycolysis would be processes parallel to glycogenolysis and glycogen synthesis, which for a long time have been known to regulate blood glucose concentration $[9,16]$.

The question arises why in former investigations differing conclusions were drawn. It is possible that estimates of glycolysis were too low because inhibition by gluconeogenic precursors was not taken into account: in experiments on interaction of glycolysis and gluconeogenesis concentrations of lactate or alanine were $20-30 \mathrm{mM}[13,14,15]$ which is one to two orders of magnitude above normal; calculations of glycolytic break-down of glucose in rat liver slices [1] and in isolated liver cells [2] were based on long incubations in which accumulation of lactate and pyruvate occurred. In the non-recirculating perfusion system used in these studies, however, inhibition by end-products was avoided by continuous wash-out.

The extracellular lactate/pyruvate ratio is used as indicator of the intracellular redox state [17]. During gluconeogenesis this ratio was 10.3 , whereas during glycolysis it went down to 3.5 (Table 2). The question arises as to how far the lactate/pyruvate ratio in glycolysis actually reflects intracellular hydrogen supply. It appears possible that steady-state conditions were not reached due to the instant removal of the end products and a different diffusability of lactate and pyruvate.

Acknowledgements. Kai Berlit is thanked for his valuable technical assistance.

\section{References}

1. Ashmore, J., Cahill, G. F., Jr., Hastings, A. B., Zottu, S.: Studies on carbohydrate metabolism in rat liver slices. VIII. Effect of ions and hormones on pathways of glucose-6phosphate metabolism. J. biol. Chem. 224, 225-235 (1957)

2. Seglen, P. O.: Autoregulation of glycolysis, respiration, gluconeogenesis and glycogen synthesis in isolated parenchymal rat liver cells under aerobic and anaerobic conditions. Biochim. biophys. Acta (Amst.) 338, 317-336 (1974)

3. Cahill, G. F., Jr., Ashmore, J., Renold, A. E. Hastings, A. B.: Blood glucose and the liver. Amer. J. Med. 26, 264-282 (1959)

5. Masoro, E. J., Chaikoff, I. L., Dauben, W. G.: Lipogenesis from glucose in the normal and liverless animal as studied with $\mathrm{C}^{14}$ labeled glucose. J. biol. Chem. 179, 1117-1125 (1949) 
6. Fröhlich, J., Hansen, W., Scholz, R.: Gluconeogenesis in rat livers perfused with nonrecirculating Krebs-Henseleit buffer. In: Isolated liver perfusion and its applications (ed. I. Bartosek) p. 205. New York: Raven Press 1973

7. Scholz, R., Hansen, W., Thurman, R. G.: Interaction of mixedfunction oxidation with biosynthetic processes. I. Inhibition of gluconeogenesis by aminopyrine in perfused rat liver. Europ. $J$. Biochem. 38, 64-72 (1973)

8. Bergmeyer, H. U.: Methoden der enzymatischen Analyse. 2nd edn. Weinheim: Verlag Chemie 1970

9. Soskin, S., Essex, H. E., Herrick, J. E., Mann, F. C.: The mechanism of regulation of the blood sugar by the liver. Amer. J. Physiol. 124, 558-567 (1938)

10. Cahill, G. F. Jr., Ashmore, J., Earle, A. S., Zottu, S.: Glucose penetration into liver. Amer. J. Physiol. 192, 491-496 (1958)

11. Hems, D. A., Whitton, P. D., Taylor, E. A.: Glycogen synthesis in the perfused liver of the starved rat. Biochem. J. 129, 529-538 (1972)

12. Walli, K., Sieber, G., Zepf, E., Schimassek, H.: Glycogen metabolism in isolated perfused rat liver. Hoppe-Seyler's Z. physiol. Chem. 355, 353-363 (1974)
13. McGraw, E. F., Peterson, M. J., Yarnell, G., Ashmore, J.: Autoregulation of glucose in the isolated perfused rat liver. Advanc. Enzyme Reg. 6, 57-65 (1968)

14. Ruderman, N. B., Herrera, M. G.: Glucose regulation of hepatic gluconeogenesis. Amer. J. Physiol. 214, 1346-1351 (1968)

15. Exton, J. H., Park, C. R.: Control of gluconeogenesis in liver. I. General features of gluconeogenesis in the perfused livers of rats. J. biol. Chem. 242, 2622-2636 (1967)

16. Bernard, C.: Neue Funktion der Leber als zuckerbereitendes Organ des Menschen und der Tiere. Würzburg: Paul Halm 1853

17. Bücher, Th., Klingenberg, M.: Wege des Wasserstoffs in der lebendigen Organisation. Angew. Chemie 70, 552-563 (1958)

Dr. W. Hansen

2. Medizinische Klinik der

Technischen Univ.

8 München 80

Ismanningerstr. 22 